\title{
LXIII. On the division of space with minimum partitional area
}

\section{Sir William Thomson}

To cite this article: Sir William Thomson (1887) LXIII. On the division of space with minimum partitional area, Philosophical Magazine Series 5, 24:151, 503-514, DOI: 10.1080/14786448708628135

To link to this article: http://dx.doi.org/10.1080/14786448708628135

曲 Published online: 29 Apr 2009.

Submit your article to this journal $₫$

Џ Article views: 32

Q View related articles $\square$

Citing articles: 224 View citing articles 두 
LXIII. On the Division of Space with Minimum Partitional Area. By Sir William Thomson*.

1. THIS problem is solved in foam, and the solution is 1 interestingly seen in the multitude of film-enclosed cells obtained by blowing air through a tube into the middle of a soap-solution in a large open vessel. I have been led to it by endeavours to understand, and to illustrate, Green's theory of "extraneous pressure" which gives, for light traversing a crystal, Fresnel's wave-surface, with Fresnel's supposition (strongly supported as it is by Stokes and Rayleigh) of velocity of propagation dependent, not on the distortionnormal, but on the line of vibration. It has been admirably illustrated, and some elements towards its solution beautifully realized in a manner convenient for study and instruction, by Plateau, in the first volume of his Statique des Liquides soumis aux seules Forces Moléculaires.

2. The general mathematical solution, as is well known, is that every interface between cells must have constant curvature throughout, and that where three or more interfaces meet in a curve or straight line their tangent-planes through any point of the line of meeting intersect at angles such that equal forces in these planes, perpendicular to their line of intersection, balance. The minimax problem would allow any number of interfaces to meet in a line; but for a pure minimum it is obvious that not more than three can meet in a line, and that therefore, in the realization by the soap-film, the equilibrium is necessarily unstable if four or more surfaces meet in a line. This theoretical conclusion is amply confirmed by observation, as we see at every intersection of films, whether interfacial in the interior of groups of soap-bubbles, large or small, or at the outer bounding-surface of a group, never more than three films, but, wherever there is intersection, always just three films, meeting in a line. The theoretical conclusion as to the angles for stable equilibrium (or pure minimum solution of the mathematical problem) therefore becomes, simply, that every angle of meeting of film-surfices is exactly $120^{\circ}$.

3. The rhombic dodecahedron is a polyhedron of plane sides between which every angle of meeting is $120^{\circ}$; and space can

* Communicated by the Author.

$\uparrow$ By "curvature" of a surface I mean sum of curratures in mutually perpendicular normal sections at any point; not Gauss's "curvatura integra," which is the product of the curvature in the two "principal normal sections," or sections of greatest and least curvature. (See 'Thomson and Tait's 'Natural Philosophy,' part i. $\$ \S 130,136$. ) 
be filled with (or divided into) equal and similar rhombic dodecahedrons. Hence it might seem that the rhombic dodecahedron is the solution of our problem for the case of all the cells equal in volume, and every part of the boundary of the group either infinitely distant from the place considered, or so adjusted as not to interfere with the homogeneousness of the interior distribution of cells. Certainly the rhombic dodecahedron is a solution of the minimax, or equilibriumproblem; and certain it is that no other plane-sided polyhedron can be a solution.

4. But it has seemed to me, on purely theoretical consideration, that the tetrahedral angles of the rhombic dodecahedron*, giving, when space is divided into such figures, twelve plane films meeting in a point (as twelve planes from the twelve edges of a cube meeting in the centre of the cube) are essentially unstable. That it is so is proved experimentally by Plateau (vol. i. $§ 182$, fig. 71) in his well-known beautiful experiment with his cubic skeleton frame dipped in soap-solution and taken out. His fig. 71 is reproduced here in fig. 1. Instead of twelve plane films stretched inwards from the twelve edges and meeting in the centre of the cube, it shows twelve films, of which eight are slightly curved and four are plane $\dagger$, stretched from the twelve edges to a small central plane quadrilateral film with equal curved edges and four angles each of $109^{\circ} 28^{\prime}$. Each of the plane films is an isosceles triangle with two equal curved sides meeting at a corner of the central curvilinear square in a plane perpendicular to its plane. It is in the plane through an edge and the centre of the cube. The angles of this plane curvilinear triangle are respectively $109^{\circ} 28^{\prime}$, at the point of meeting of the two curvilinear sides : and each of the two others half of this, or $54^{\circ} 44^{\prime}$.

5. I find that by blowing gently upon the Plateau cube into any one of the square apertures through which the little central quadrilateral film is seen as a line, this film is caused

* The rhombic dodecahedron has six tetrahedral angles and eight trihedral angles. At each tetrahedral angle the plane faces cut one another successively at $120^{\circ}$, while each is perpendicular to the one remote from it ; and the angle between successive edges is $\cos ^{-1} \frac{1}{3}$, or $70^{\circ} 32^{\prime}$. The obtuse angles $\left(109^{\circ} 28^{\prime}\right.$ ) of the rhombs meet in the trihedral angles of the solid figure. The whole figure may be regarded as composed of six square pyramids, each with its alternate slant faces perpendicular to one another, placed on six squares forming the sides of a cube. The long diagonal of each rhombic face thus made up of two sides of pyramids conterminous in the short diagonal, is $\sqrt{2}$ times the short diagonal.

$\dagger$ I see it inadvertently stated by Plateau that all the twelve films are "légèrement courbées." 
to contract. If I stop blowing before it contracts to a point, it springs back to its primitive size and shape. If I blow still very gently but for a little more time, the quadrilateral contracts to a point, and the twelve films meeting in it immediately draw out a fresh little quadrilateral film similar to the former, but in a plane perpendicular to its plane and to the direction of the blast. Thus, again and again, may the films be transformed so as to render the little central curvilinear square parallel to one or other of the three pairs of square apertures of the cubic frame. Thus we see that the twelve plane films meeting in the centre of the cube is a configuration of unstable equilibrium which may be fallen from in three different ways.

b. Suppose now space to be filled with equal and similar ideal rhombic

Fig. 1.

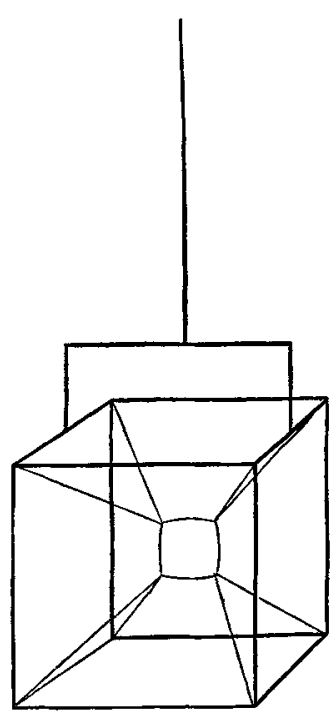
dodecahedrons. Draw the short dis.gonal of every rhombic face, and fix a real wire (infinitely thin and perfectly stiff) along each. This fills space with Plateau cubic frames. Fix now, ideally, a very small rigid globe at each of the points of space occupied by tetrahedral angles of the dodecahedrons, and let the faces of the dodecahedrons be realized by soap-films. They will be in stable equilibrium, because of the little fixed globes; and the equilibrium would be stable without the rigid diagonals which we require only to help the imagination in what follows. Let an exceedingly small force, like gravity*, act on all the films everywhere perpendicularly to one set of parallel faces of the cubes. If this force is small enough it will not tear away the films from the globes; it will only produce a very slight bending from the plane rhombic shape of each film. Now annul the little globes. The films will instantly jump (each set of twelve which meet in a point) into the Plateau configuration (fig. 1), with the little curve-edged square in the plane perpendicular to the determining force, which may now be annulled, as we no longer require it. The rigid edges of the cubes may also be now annulled, as we have done with them also ; because each is (as we see by symmetry) pulled with equal forces in opposite directions, and therefore is not required for the equi-

* To do for every point of meeting of twelve films what is done by blowing in the experiment of $\$ 5$. 
librium, and it is clear that the equilibrium is stable without them*.

* The corresponding two-dimensional problem is much more easily imagined; and may probably be realized by aid of moderately simple appliances.

Between a level surface of soap-solution and a horizontal plate of glass fixed at a centimetre or two above it, imagine vertical film-partitions to be placed along the sides of the squares indicated in the drawing (fig, 2):

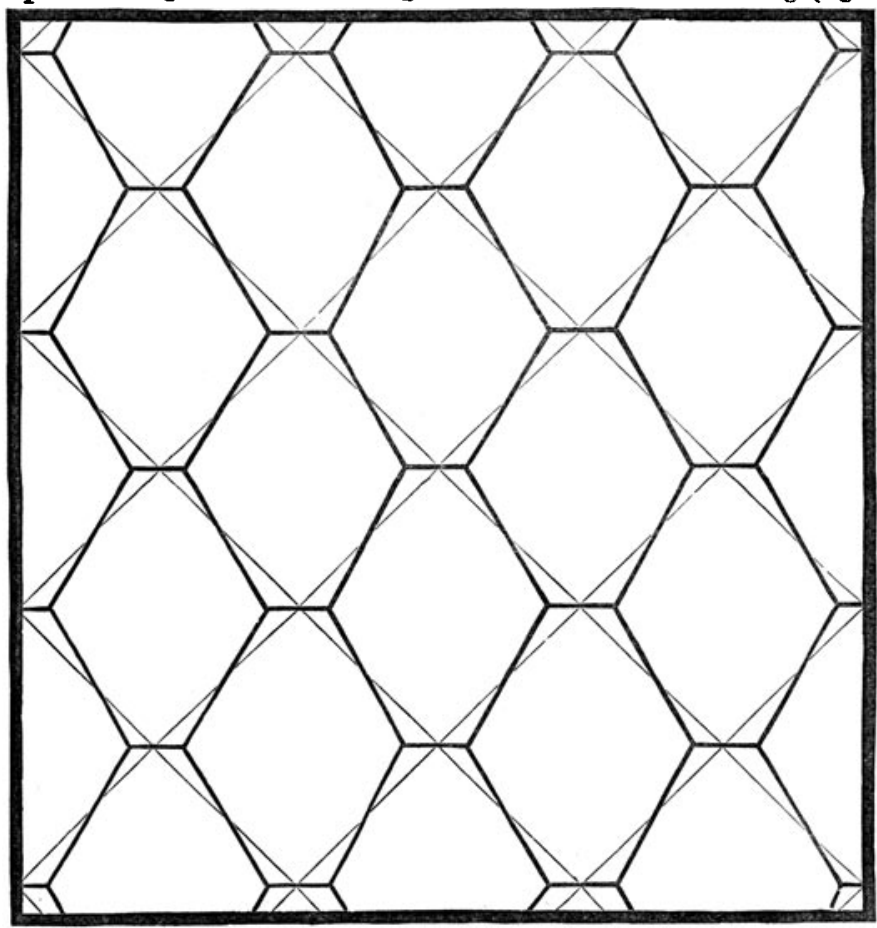

Fig. 2.

these will rest in stable equilibrium if thick enough wires are fixed vertically through the corners of the squares. Now draw away these wires downwards into the liquid: the equilibrium in the square formation becomes nnstable, and the films instantly run into the hexagonal formation shown in the diagram; provided the square of glass is provided with vertical walls (for which slips of wood are convenient), as shown in plan by the black border of the diagram. These walls are necessary to maintain the inequality of pull in different directions which the inequality of the sides of the hexagons implies. By inspection of the diagram we see that the pull is $\mathrm{T} / a$ per unit area on either of the pair of vertical walls which are perpendicular to the short sides of the hexagons; and on either of the other pair of walls $2 \cos 30^{\circ} \times \mathrm{T} / a$; where $\mathrm{T}$ denotes the pull of the film per unit breadth, and $a$ the side of a square in the original formation. Hence the ratio of the pulls per unit of area in the two principal directions is as 1 to $] \cdot 732$. 
7. We have now space divided into equal and similar tetrakaidecahedral cells by the soap-film ; each bounded by

(1) Two small plane quadrilaterals parallel to one another ;

(2) Four large plane quadrilaterals in planes perpendicular to the diagonals of the small ones ;

(3) Eight non-plane hexagons, each with two ed ges common with the small quadrilaterals, and four edges common with the large quadrilaterals.

The films seen in the Plateau cube show one complete small quadrilateral, four halves of four of the large quadrilaterals, and eight halves of eight of the hexagons, belonging. to six contiguous cells; all mathematically correct in every part (supposing the film and the cube-frame to be infinitely thin). Thus we see all the elements required for an exact construction of the complete tetrakaidecahedron. By making a clay model of what we actually see, we have only to complete a symmetrical figure by symmetrically completing each half-quadrilateral and each half-hexagon, and putting the twelve properly together, with the complete small quadrilateral, and another like it as the far side of the 14-faced figure. We thus have a correct solid model.

8. Consider now a cubic portion of space containing a large number of such cells, and of course a large but a comparatively small number of partial cells next the boundary. Wherever the boundary is cut by film, fix stiff wire; and remove all the film from outside, leaving the cubic space divided stably into cells by films held out against their tension by the wire network thus fixed in the faces of the cube. If the cube is chosen with its six faces parallel to the three pairs of quadrilateral films, it is clear that the resultant of the whole pull of film on each face will be perpendicular to the face, and that the resultant pulls on the two pairs of faces parallel to pairs of the greater quadrilaterals are equal to one another and less than the resultant pull on the pair of faces parallel to the smaller quadrilaterals. Let now the lastmentioned pair of faces of the cube be allowed to yield to the pull inwards, while the other two pairs are dragged outwards against the pulls on them, so as to keep the enclosed volume unchanged; and let the wirework fixture on the faces be properly altered, shrunk on two pairs of faces, and extended on the other pair of faces, of the cube, which now becomes a square cage with distance between floor and ceiling less than the side of the square. Let the exact configuration of the wire everywhere be always so adjusted that the cells throughout the interior remain, in their altered configuration, equal and similar to one another. We may thus diminish, and if we 
please annul, the difference of pull per unit area on the three pairs of sides of the cage. The respective shrinkage-ratio and extension-ratio, to exactly equalize the pulls per unit area on the three principal planes, (and therefore on all planes), are $2^{-\frac{1}{3}}, 2^{\frac{1}{6}}, 2^{\frac{1}{6}}$, as is easily seen from what follows.

9. While the equalization of pulls in the three principal directions is thus produced, work is done by the film on the moving wire-work of the cage, and the total area of film is diminished by an amount equal to $W / T$, if $W$ denote the whole work done, and $\mathrm{T}$ the pull of the film per unit breadth. The change of shape of the cage being supposed to be performed infinitely slowly, so that the film is always in equilibrium throughout, the total area is at each instant a minimum, subject to the conditions

(1) That the volume of each cell is the given amount;

(2) That every part of the wire has area edged by it; and

(3) That no portion of area has any free edge.

10. Consider now the figure of the cell (still of course a tetrakaidecahedron) when the pulls in the three principal directions are equalized, as described in $\S 8$. It must be perfectly isotropic in respect to these three directions. Hence the pair of small quadrilaterals must have become enlarged to equality with the two pairs of large ones, which must have become smaller in the deformational process described in $\S 8$. Of each hexagon three edges coincide with edges of quadrilateral faces of one cell; and each of the three others coincides with edges of three of the quadrilaterals of one of the contiguous cells. Hence the 36 edges of the isotropic tetrakaidecahedron are equal and similar plane arcs ; each of course symmetrical about its middle point. Every angle of meeting of edges is essentially $109^{\circ} 28^{\prime}$ (to make trihedral angles between tangent planes of the films meeting at $120^{\circ}$ ). Symmetry shows that the quadrilaterals are still plane figures; and therefore, as each angle of each of them is $109^{\circ} 28^{\prime}$, the change of direction from end to end of each areedge is $19^{\circ} 28^{\prime}$. Hence each would be simply a circular arc of $19^{\circ} 28^{\prime}$, if its curvature were equal throughout; and it seems from the complete mathematical investigation of $\$ \S 16$, 17,18 below, that it is nearly so, but not exactly so even to a first approximation.

Of the three films which meet in each edge, in three adjacent cells, one is quadrilateral and two are hexagonal.

11. By symmetry we see that there are three straight lines in each (non-plane) hexagonal film, being its three' long diagonals; and that these three lines, and therefore the six angular points of the hexagon, are all in one plane. The arcs 
composing its edges are not in this plane, but in planes making, as we shall see $(\$ 12)$, angles of $54^{\circ} 44^{\prime}$ with it. For three edges of each hexagon, the planes of the ares bisect the angle of $109^{\circ} 28^{\prime}$ between the planes of the six corners of contiguous hexagons; and for the other three edges are inclined on the outside of its plane of corners, at angles equal to the supplements of the angles of $125^{\circ} 16^{\prime}$ between its plane of corners and the planes of contiguous quadrilaterals.

12. The planes of corners of the eight hexagons constitute the faces of an octahedron which we see, by symmetry, must be a regular octahedron (eight equilateral triangles in planes inclined $109^{\circ} 28^{\prime}$ at every common edge). Hence these planes, and the planes of the six quadrilaterals, constitute a plane-faced tetrakaidecahedron obtained by truncating the six corners * of a regular octahedron each to such a depth as to reduce its eight original (equilateral triangular) faces to equilateral equiangular hexagons. An orthogonal projection of this figure is shown in fig. 3 . It is to be remarked that space can be filled with such figures. For brevity we shall call it a plane-faced isotropic tetrakaidecahedron.

13. Given a model of the plane-faced isotropic tetrakaidecahedron, it is easy to construct approximately a model of the minimal tetrakaidecahedron, thus :-Place on each of the six square faces a thin plane disk having the proper curved ares of $19^{\circ} 28^{\prime}$ for its edges. Draw the three long diagonals of each hexagonal face. Fill up by little pieces of wood, properly cut, the three sectors of $60^{\circ}$ from the centre to the overhanging

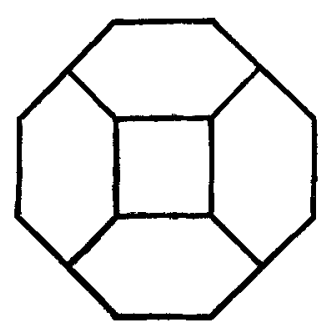

Fig. 3.

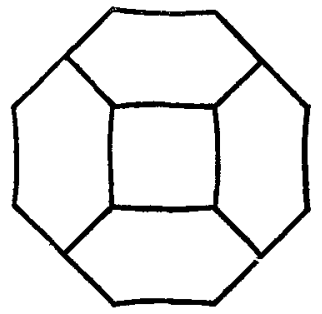

Fig. 4.

edges of the adjacent quadrilaterals. Hollow out symmetrically the other three sectors, and the thing is done. The result is shown in orthogonal projection, so far as the edges are concerned, in fig. 4 ; and as the orthogonal projections are equal and similar on three planes at right angles to one another,

* This figure (but with probably indefinite extents of the truncation) is given in books on mineralogy as representing a natural crystal of red oxide of copper. 
this diagram suffices to allow a perspective drawing from any point of view to be made by "descriptive geometry."

14. No shading could show satisfactorily the delicate curvature of the hexagonal faces, though it may be fairly well seen on the solid model made as described in $\S 12$. But it is shown beautifully, and illustrated in great perfection, by making a skeleton model of 36 wire arcs for the 36 edges of the complete figure, and dipping it in soap solution to fill the faces with film, which is easily done for all the faces but one. The curvature of the hexagonal film on the two sides of the plane of its six long diagonals is beautifully shown by reflected light. I have made these 36 arcs by cutting two circles, 6 inches diameter, of stiff wire, each into 18 parts of $20^{\circ}$ (near enough to $19^{\circ} 28^{\prime}$ ). It is easy to put them together in proper positions and solder the corners, by aid of simple devices for holding the ends of the three arcs together in proper positions during the soldering. The circular curvature of the arcs is not mathematically correct, but the error due to it is, no doubt, hardly perceptible to the eye.

15. But the true form of the curved edges of the quadrilateral plane films, and of the non-plane sufaces of the hexagonal films, may be shown with mathematical exactness by taking, instead of Plateau's skeleton cube, a skeleton square cage with four parallel edges each 4 centimetres long: and the other eight, constituting the edges of two squares each

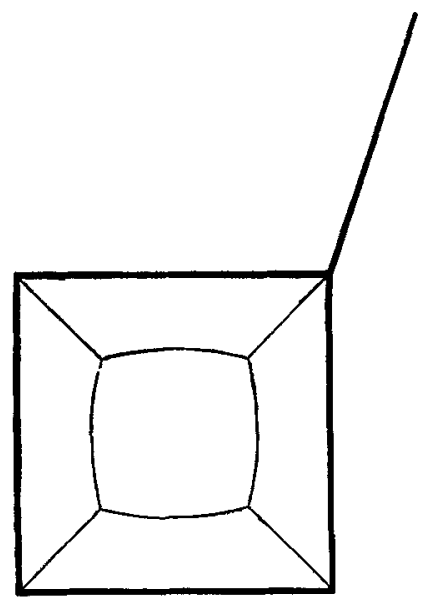

Fig. 5.

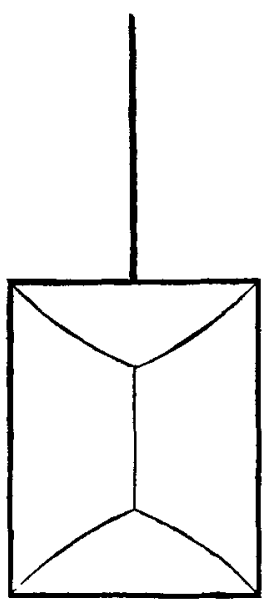

Fig. 6.

$\sqrt{ } 2$ times as long, or 5.66 centim. Dipped in soap-solution and taken out it always unambiguously gives the central 
quadrilateral in the plane perpendicular to the four short edges. It shows with mathematical accuracy (if we suppose the wire edges infinitely thin) a complete quadrilateral, four half-quadrilaterals, and four half-hexagons of the minimal tetrakaidecahedron. The two principal views are represented in figs. 5 and 6.

16. The mathematical problem of calculating the forms of the plane arc-edges, and of the curved surface of the hexagonal faces, is easily carried out to any degree of approximation that may be desired; though it would be very laborious, and not worth the trouble, to do so further than a first approximation, as given in $\$ 17$ below. But first let us state the rigorous mathematical problem; which by symmetry becomes narrowed to the consideration of a $60^{\circ}$ sector $\mathrm{BC} \mathrm{B}^{\prime}$ of our non-plane hexagon, bounded by straight lines $\mathrm{C} \mathrm{B}, \mathrm{CB}^{\prime}$. and a slightly curved edge $\mathrm{BE} \mathrm{B}^{\prime}$, in a plane, $\mathrm{Q}$, through

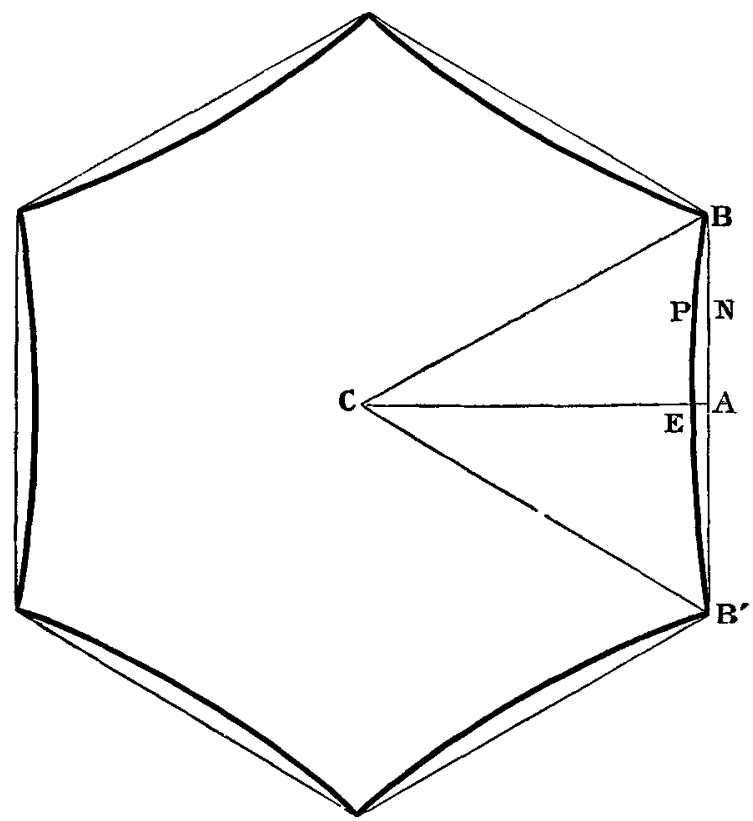

Fig. 7.

$B B^{\prime}$, inclined to the plane $B C B^{\prime}$ at an angle of $\tan ^{-1} \sqrt{ } 2$, or $54^{\circ} 44^{\prime}$. The plane of the curved edge I call $Q$, because it is the plane of the contiguous quadrilateral. The mathematical problem to be solved is to find the surface of zero curvature edged by $\mathrm{B} \mathrm{C} \mathrm{B'} \mathrm{and} \mathrm{cutting} \mathrm{at} 120^{\circ}$ the plane $\mathrm{Q}$ all along the intersectional curve (fig. 7). It is obvious that this problem is 
determinate and has only one solution. Taking $\mathrm{CA}$ for axis of $x$; and $z$ perpendicular to the plane $\mathrm{BCB}^{\prime}$ : and regarding $z$ as a function of $x, y$, to be determined for finding the form of the surface, we have, as the analytical expression of the conditions

$$
\frac{d^{2} z}{d x^{2}}\left(1+\frac{d z^{2}}{d y^{2}}\right)-2 \frac{d z}{d x} \frac{d z}{d y} \frac{d^{2} z}{d x d y}+\frac{d^{2} z}{d y^{2}}\left(1+\frac{d z^{2}}{d x^{2}}\right)=0
$$

and

$$
\left.\begin{array}{r}
\left(1+\frac{d z^{2}}{d x^{2}}+\frac{d z^{2}}{d y^{2}}\right)^{-\frac{1}{2}}\left(\sqrt{3}-\frac{d z}{d x} \sqrt{ } \frac{2}{3}\right)=\frac{1}{2} \\
\text { when } z=(a-x) \sqrt{ } 2
\end{array}\right\}
$$

17. The required surface deviates so little from the plane $\mathrm{BCB}^{\prime}$ that we get a good approximation to its shape by neglecting $d z^{2} / d x^{2}, d z / d x \cdot d z / d y$, and $d z^{2} / d y^{2}$, in (1) and (2), which thus become

and

$$
\nabla^{2} z=0 \text {. . . . . . . . }
$$

$$
\frac{d z}{d x}=\frac{\sqrt{ } 2}{2}-\sqrt{ } \frac{3}{8}=\cdot 094735, \text { when } x=a-z / \sqrt{ } 2, .
$$

$\nabla^{2}$ denoting $(d / d x)^{2}+(d / d y)^{2}$. The general solution of (3), in polar coordinates $(r, \phi)$ for the plane $(x, y)$, is

$$
\Sigma(\mathrm{A} \cos m \phi+\mathrm{B} \sin m \phi) r^{m}, \quad . \quad \text {. . . }
$$

where A, B, and $m$ are arbitrary constants. The symmetry of our problem requires $\mathrm{B}=0$, and $m=3 .(2 i+1)$, where $i$ is any integer. We shall not take more than two terms. It seems not probable that advantage could be gained by taking more than two, unless we also fall back on the rigorous equations (1) and (2), keeping $d z^{2} / d x^{2} \& c$. in the account, which would require each coefficient $A$ to be not rigorously constant but a function of $r$. At all events we satisfy ourselves with the approximation yielded by two terms, and assume

$$
z=\mathrm{A} r^{3} \cos 3 \phi+\mathrm{A}^{\prime} r^{9} \cos 9 \phi \quad \text {. . . }
$$

with two coefficients $A, A^{\prime}$ to be determined so as to satisfy (4) for two points of the curved edge, which, for simplicity, we shall take as its middle, $\mathrm{E}(\phi=0)$; and end, $\mathrm{B}\left(\phi=30^{\circ}\right)$. Now remark that, as $z$ is small, even at $E$, where it is greatest, we have, in (4), $x \fallingdotseq a$ or $r \fallingdotseq a \sec \phi$. Thus, and substituting for $d z / d x$ its expression in polar $(r, \phi)$ coordinates, which is

$$
\frac{d z}{d x}=\frac{d z}{d r} \cos \phi-\frac{d z}{r d \phi} \sin \phi . . . .
$$


we find, from (4) with (6),

(by case $\phi=0) \quad \mathrm{A}+3 a^{6} \mathrm{~A}^{\prime}=\cdot 031578 a^{-2}$. . . (8), (and by case $\left.\phi=30^{\circ}\right) A-{ }_{9}^{64} a^{6} \mathrm{~A}^{\prime}=\cdot 031578 \cdot \frac{3}{2} \cdot a^{-2} \quad$. (9); whence

$$
\begin{aligned}
\mathrm{A}^{\prime}=-\frac{1}{2} \cdot \frac{9}{91} \times \cdot 031578 . a^{-8} & =-9 \times \cdot 0001735 \cdot a^{-8} \\
& =-\cdot 001561 \cdot a^{-8} \\
\mathrm{~A}=\frac{1}{2}\left(3-\frac{64}{91}\right) \times \cdot 031578 . a^{-2} & =209 \times \cdot 0001735 \cdot a^{-2} \\
& =036261 \cdot a^{-} ;
\end{aligned}
$$

and for required equation of the surface we have (taking $a=1$ for brevity)

$$
\left.\begin{array}{rl}
z & =\cdot 03626 \cdot r^{3} \cos 3 \phi-\cdot 001561 r^{9} \cos 9 \phi \\
& =\cdot 03626 \cdot r^{3}\left(\cos 3 \phi-\cdot 043 \cdot r^{6} \cos 9 \phi\right)
\end{array}\right\} .
$$

18. To find the equation of the curved edge $\mathrm{BEB}^{\prime}$, take, as in (4),

$$
x=1-z \sqrt{ } / 2=1-\xi \text {, where } \xi \text { denotes } z \sqrt{ } 2 \ldots \text {. (11). }
$$

Substituting in this, for $z$, its value by (10), with for $r$ its approximate value $\sec \phi$, we find

$$
\xi=\frac{1}{\sqrt{ } 2}\left({ }^{\circ} 03626 \sec ^{3} \phi \cos 3 \phi-\cdot 001561 \sec ^{9} \phi \cos 9 \phi\right) \text {. }
$$

as the equation of the orthogonal projection of the edge, on the plane $\mathrm{BCB}^{\prime}$, with

$$
\mathrm{AN}=y=\tan \phi ; \text { and } \mathrm{NP}=\xi \quad . \quad \text {. (13). }
$$

The diagram was drawn to represent this projection roughly, as a circular arc, the projection on $\mathrm{BCB}^{\prime}$ of the circular arc of $20^{\circ}$ in the plane $\mathrm{Q}$, which, before making the mathematical investigation, I had taken as the form of the arc-edges of the plane quadrilaterals. This would give $1 / 35$ of $\mathrm{CA}$, for the sagitta, $\mathrm{AE}$; which we now see is somewhat too great. The equation (12), with $y=0$, gives for the sagitta

$$
\mathrm{AE}=\cdot 0245 \times \mathrm{CA} \text {. . . . . (14), }
$$

or, say, $1 / 41$ of $\mathrm{CA}$. The curvature of the projection at any point is to be found by expressing $\sec ^{3} \phi \cos 3 \phi$ and $\sec ^{9} \phi \cos 9 \phi$ in terms of $y=\tan \phi$ and taking $d^{2} / d y^{2}$ of the result.

By taking $\sqrt{ }(3 / 2)$ instead of $\sqrt{ }(1 / 2)$ in (12), we have the equation of the are itself in the plane $Q$.

19. To judge of the accuracy of our approximation, let us find the greatest inclination of the surface to the plane $\mathrm{BCB}^{\prime}$. For the tangent of the inclination at $(r, \phi)$ we have $\left(\frac{d z^{2}}{d r^{2}}+\frac{d z^{2}}{r^{2} d \phi^{2}}\right)^{\frac{1}{2}}=\cdot 1088 . r^{2}\left(1-2 \times \cdot 129 \cdot r^{6} \sec 6 \phi+\cdot 129^{2} r^{12}\right)^{\frac{1}{2}}$

\footnotetext{
Phil. Mag. S. 5. Vol. 24. No. 151. Dec. 1887. $2 \mathrm{M}$
} 
The greatest values of this will be found at the curved bounding edge, for which $r \fallingdotseq \sec \phi$. Thus we find

$$
\begin{aligned}
& \left(1+\frac{d z^{2}}{d r^{2}}+\frac{1}{r^{2}} \frac{d z^{2}}{d \phi^{2}}\right)^{\frac{1}{2}} \\
& \left\{\begin{array}{l}
=0948, \text { and therefore inclination }=5^{\circ} 25^{\prime} \text { at } \mathrm{E} \\
=\cdot 1892, \quad,, \quad, \quad=10^{\circ} 43^{\prime} \text { at } \mathrm{B}
\end{array}\right\}
\end{aligned}
$$

Hence we see that the inaccuracy due to neglecting the square of the tangent of the inclination in the mathematical work cannot be large. The exact value of the inclination at $\mathrm{E}$ is $\tan ^{-1}(-\sqrt{ } 2)-120^{\circ}$, or $5^{\circ} 16^{\prime}$, which is less by $9^{\prime}$ than its value by $(16)$.

LXIV. On the Application of the Deci-ampere or the Centiampere Balance to the Determination of the Electromotive Forces of Voltaic Cells. By Sir William Thomson, F.R.S.*

THE method described in this paper for the determination, 1 in absolute measure, of the electromotive forces of voltaic cells, consists in the use of one of my standard amperebalances instead of the tangent-galvanometer in the method given in the following statement, which I quote from Kohlrausch's 'Physical Measurements,' pp. 223, 224, 230:-“"The only methods applicable to inconstant elements, of which the electromotive force varies with the current-strength, is to bring the current to zero by opposing an equal electromotive force. Poggendorff's method, which is very convenient, as it involves no measurement of internal resistance, requires the use of a galvanoscope, G, a galvanometer, T, and a rheostat, R, and, in addition, that of an auxiliary battery, $\mathrm{S}$, of constant electromotive force, greater than either of those which are to be compared. The arrangement of the experiment is shown in the figure. In the left division of the circuit are the galvanoscope $G$, and the electromotive force $E$ to be measured; in the right, the auxiliary battery $\mathrm{S}$ and the galvanometer $\mathrm{T}$. $\mathrm{E}$ and $\mathrm{S}$ are so,placed that their similar poles are turned towards each other. In the middle part of the cir-

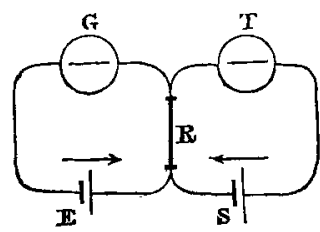

Fig. 1. cuit, which is common to both batteries, is the rheostat $R$.

"As much rheostat resistance W must now be intercalated

* Communicated by the Author, being a paper read at the Manchester Meeting of the British Association, with additions. 